\title{
Isotope solar modulation with the PAMELA experiment.
}

\author{
Riccardo Munini $^{\star a, b}$, O. P. M. Aslam ${ }^{c}$, D. Bisschoff ${ }^{c}$, M. Boezio ${ }^{a, b}$, A. Lenni ${ }^{a, b, d}$, W. \\ Menn $^{e}$, N. Marcelli ${ }^{f}$, M. S. Potgieter, On behalf of the PAMELA collaboration \\ ${ }^{a}$ INFN, Sezione di Trieste, I-34149 Trieste, Italy \\ ${ }^{b}$ IFPU - Institute for Fundamental Physics of the Universe, I-34014, Trieste, Italy \\ ${ }^{c}$ Centre for Space Reseach, North-West University, , 2520 Potchefstroom, South Africa \\ ${ }^{d}$ University of Trieste, Department of Physics, I-34147 Trieste, Italy \\ e Universitat Siegen, Department of Physics, D-57068 Siegen, Germany \\ ${ }^{f}$ INFN, Sezione di Roma "Tor Vergata”, I-00133 Rome, Italy \\ E-mail: riccardo.munini@ts.infn.it, aslamklr2003@gmail.com, \\ driaanb@gmail.com, mirko.boezio@ts.infn.it, alex.lenni@ts.infn.it, \\ menn@pamela.physik.uni-siegen.de,nadir.marcellieroma2.infn.it, \\ marius.potgieter@nwu.ac.za
}

\begin{abstract}
The satellite-borne PAMELA experiment was launched on the 15th June 2006 from the Baikonur cosmodrome. Since then and until January 2016 PAMELA made high-precision measurements of the charged component of cosmic-rays over a wide energy range. Because of its long-duration operation, PAMELA represents an ideal detector for cosmic-ray solar modulation studies. The PAMELA collaboration already published time-dependent proton, helium and electron spectra as well as the positron to electron ratio over ten years of data taking. These results are fundamental to improve and refine propagation models the propagation models of cosmic rays through the heliosphere.

Here, the yearly average spectra over the 23rd solar minimum (July 2006 - January 2009) until the middle of the $24 \mathrm{rd}$ solar maximum (December 2015) for ${ }^{1} \mathrm{H},{ }^{2} \mathrm{H},{ }^{3} \mathrm{He}$ and ${ }^{4} \mathrm{He}$ are presented. The isotopic composition was measured between 0.1 and $1.1 \mathrm{GeV} / \mathrm{n}$ using two different detector systems. These spectra show an increasing trend during the solar minimum period with a decrease as the solar maximum is approached. The time-dependent ratio of these isotopes is also presented. From a solar modulation point of view, a non constant ratio is typically caused by their different masses (related to their rigidity) and also the different shapes of the respective local interstellar spectra. Apart from these effects, it is worthwhile to look deeper for more fundamental causes related to their propagation and modulation mechanisms.
\end{abstract}

36th International Cosmic Ray Conference -ICRC2019-

July 24th - August 1st, 2019

Madison, WI, U.S.A.

\footnotetext{
* Speaker.
} 


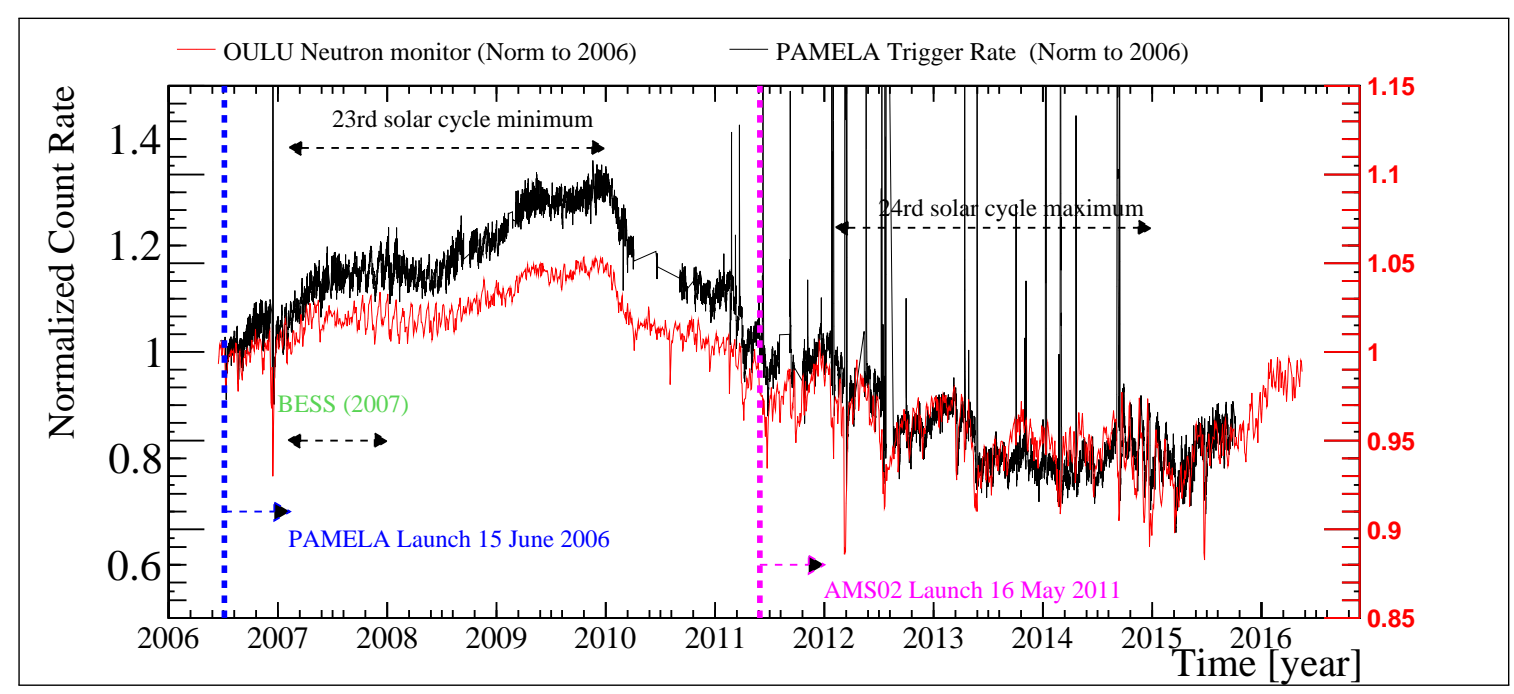

Figure 1: Trigger rate of the PAMELA apparatus from July 2006 to January 2016 (black line) compared with OULU neutron monitor intensity (red line). The two profiles are normalized to July 2006.

\section{Introduction}

The ${ }^{2} \mathrm{H}$ and ${ }^{3} \mathrm{He}$ isotopes in cosmic rays (CRs) are mostly of secondary origin, resulting from the nuclear interactions of primary $\mathrm{CR}$ protons and ${ }^{4} \mathrm{He}$ with the interstellar medium. The spectral shape and composition of the secondary isotopes are therefore completely determined by the source spectrum of the parent elements and by the propagation process. Measurements of the secondary isotopes spectra are then a powerful tool to constrain the parameters of the galactic propagation models. The PAMELA collaboration already published result on the ${ }^{1} \mathrm{H},{ }^{2} \mathrm{H},{ }^{3} \mathrm{He}$ and ${ }^{4} \mathrm{He}$ isotopes between $\sim 0.1$ and $1.1 \mathrm{GeV} / \mathrm{n}$ with data collected between July 2006 and December 2007 [6].

However, below few tens of GeV/n, CRs are heavily affected by the solar modulation which reduce their intensity and modify their spectral shape with respect to the Local Interstellar Spectrum (LIS), i.e. the spectrum of CRs outside the Heliosphere. Moreover, the solar modulation introduce a time dependence in the CR fluxes measured inside the Heliosphere. During solar maxima the intensity of CRs is lower with respect to periods of solar minima as a consequence of the stronger heliospheric magnetic field as a consequence of increased solar activity with a subsequent more turbulent solar wind and imbedded heliospheric magnetic field, and a more wavy current sheet.. This effect is illustrated in Figure 1 which shows the trigger rate of the PAMELA experiment compared with the neutron intensity measured by the OULU neutron monitor. The maximum intensity is reached around late 2009 at the end of the $23^{\text {rd }}$ solar minimum while the lower intensity correspond to the middle of the $24^{\text {th }}$ solar maximum.

In this work, the isotope analysis has been extended to data collected with PAMELA up to September 2014 in order to study the time evolution of the fluxes. Precise measurements of the time dependent isotopes spectra provide important informations about their propagation mechanism through the heliosphere emphasizing possible different modulation caused by their masses or the different shapes of the respective LIS. A 3D numerical model which solves the Parker equation, e.g. see $[1,2]$, was already calibrated and tested with the PAMELA time dependent measurements 


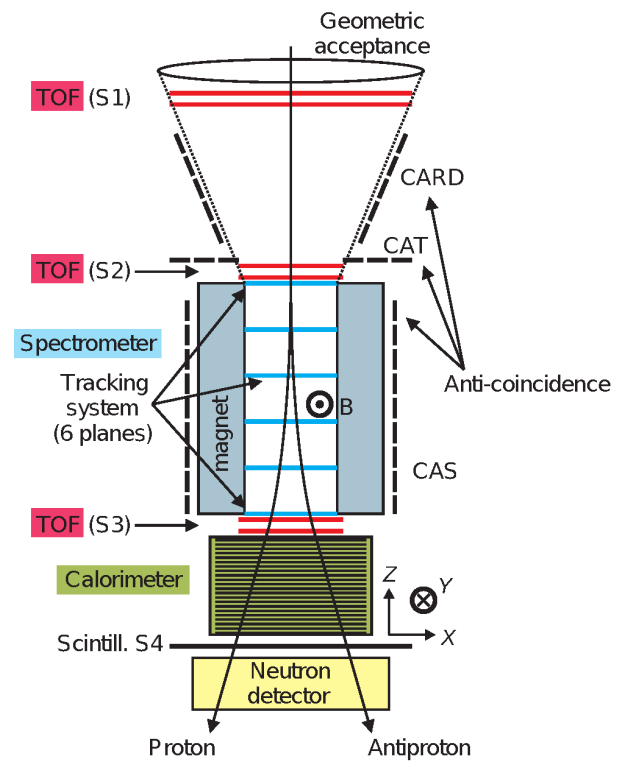

Figure 2: Schematic view of the PAMELA apparatus.

on protons [3] [4] and electrons [5], and will be used to interpret the isotope modulation.

In this contribution the analysis performed to obtain the isotope fluxes will be discussed. In order to reach the best time resolution keeping a good statistics the yearly average fluxes were measured. The Preliminary results will be presented at the conference.

\section{The PAMELA apparatus}

The PAMELA instrument [8], launched on the 15th June 2006 from the Baikonur cosmodrome in Kazakistan, was installed on board of the Russian satellite Resurs DK1 and measured the cosmic radiation untill January 2016. The PAMELA mission covers almost an entire solar cycle, from the middle of the $23^{\text {rd }}$ solar minimum to the middle of the $24^{\text {th }}$ solar maximum. Hence, the data collected during this time represents an excellent opportunity to study the time dependent evolution of the low energy cosmic radiation due to solar modulation.

A schematic view of the PAMELA instrument is shown in Figure 2. The core of the apparatus is a magnetic cavity equipped with six planes of double face microstrip silicon detector. The tracker is used to measured the rigidity $\mathrm{R}$ of the incoming particle defined as $R=p c / Z e$ where $p$ is the particle momentum, $c$ the speed of light and $Z e$ the electric charge. The tracker planes perform also twelve independent energy losses measurements $\mathrm{dE} / \mathrm{dx}$, which are extensively used in the isotopes analysis. A time of flight (ToF) system is composed by twelve planes of plastic scintillator and performs a velocity measurement, twelve independent $\mathrm{dE} / \mathrm{dx}$ measurements and trigger the data acquisition. Finally an electromagnetic calorimeter made of 44 single-sided silicon sensor planes detector interleaved with 22 planes of tungsten, mainly used for hadron-lepton separation, performs also multiple energy losses measurements. The high redundancy of the PAMELA subdetectors and the multiple independent $\mathrm{dE} / \mathrm{dx}$ measurements allows to perform a precise study of the isotopes composition of CR hydrogen and helium. 


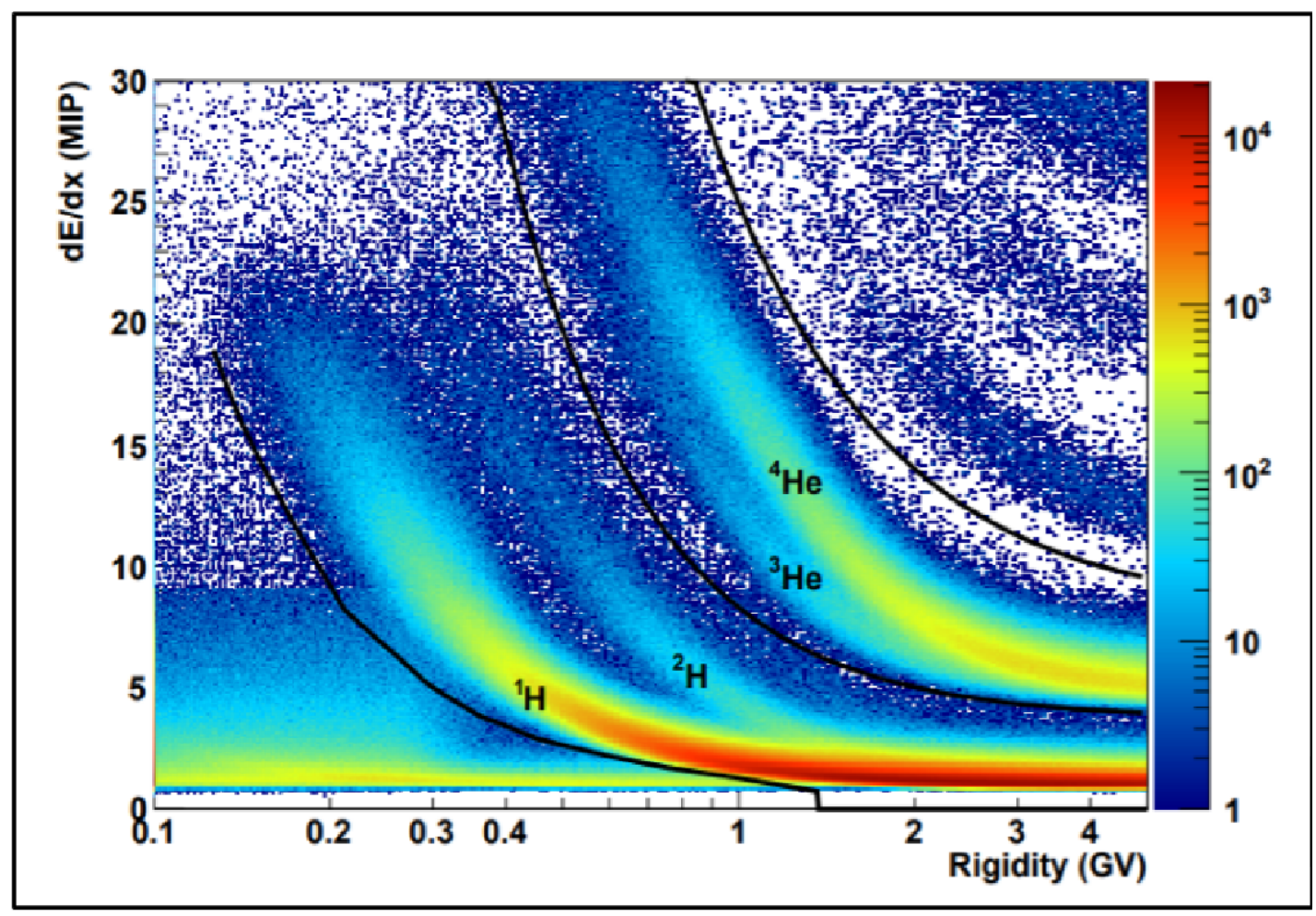

Figure 3: Mean ionization energy losses $\mathrm{dE} / \mathrm{dx}$ in the trackers planes as a function of the rigidity. The black lines represent the selection for $\mathrm{Z}=1$ or $\mathrm{Z}=2$ nuclei.

\section{Data Analysis}

\subsection{Event Selection}

Each triggered event had to fulfill several criteria to be used for further analysis. The requirements are extensively described in [6]: the tracker was used to select a sample of events with a single track reconstructed at least $1.5 \mathrm{~mm}$ away from the magnet wall. The events must have a lever-arm of at least 4 silicon planes and a minimum of 3 hits on the bending $x$-view and on nonbending $y$-view respectively. Bad quality tracks were rejected imposing a cut on the $\chi^{2}$ of the fitting algorithm.

From these events two samples of $\mathrm{Z}=1$ and $\mathrm{Z}=2$ particles were selected using the mean energy losses obtained by the twelve tracker planes, see Figure 3. The black solid lines represent the cuts defined to select hydrogen and helium. These cuts ensure a negligible contamination, of the order of $10^{-3}-10^{-4}$, of $Z=2$ particles in the $Z=1$ sample and vice-versa. This contamination was calculated applying the tracker isotope selection for $Z=1$ and $Z=2$ respectively to a sample of $Z=2$ or $\mathrm{Z}=1$ particles selected with the $\mathrm{ToF} \mathrm{dE} / \mathrm{dx}$.

\subsection{Isotope Selection}

From these samples of events the isotopic separation was performed using two different techniques: 

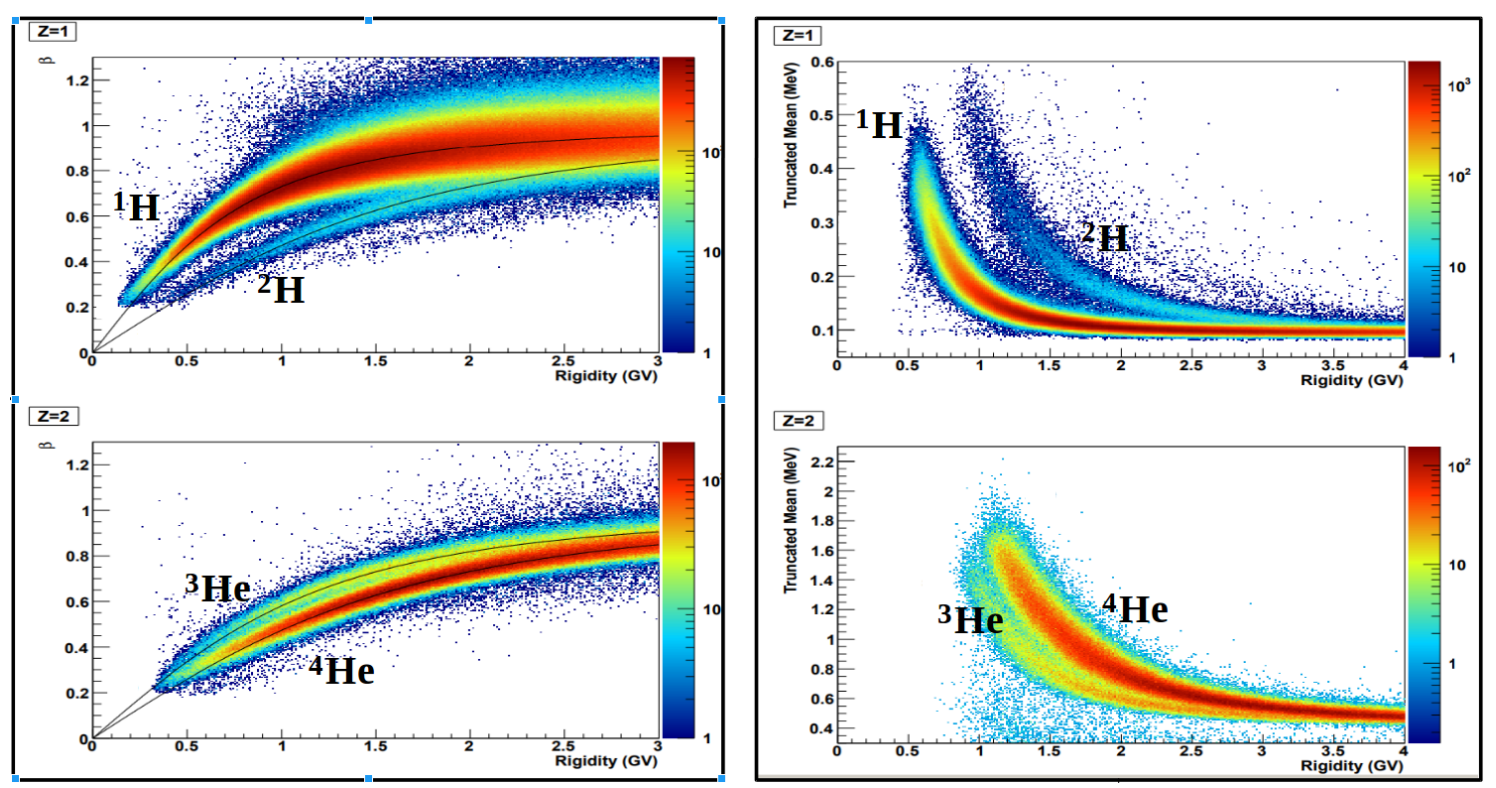

Figure 4: Left panels: $\beta$ distribution as a function of the rigidity for $\mathrm{Z}=1$ particles (upper panel) and $\mathrm{Z}=2$ particles (lower panel). The black lines represent the expected theoretical $\beta$ values for the different isotopes. Right panels: $\mathrm{dE} / \mathrm{dx}$ measured in the calorimeter as a function of the rigidity for $\mathrm{Z}=1$ particles (upper panel) and $\mathrm{Z}=2$ particles (lower panel).

1. Isotope separation using ToF versus rigidity: For the ToF analysis, the $\beta$ ( $\beta=v / c$ with $v$ the velocity of the particle) provided by the timing measurement was used. Figure 4 left panel shows the $\beta$ distribution as a function of rigidity for the sample of $Z=1$ (top panel) and $Z=2$ (bottom panel) particle selected with the tracker. The black lines in the figure represent the expectations for each isotope.

2. Isotope separation using multiple $\mathrm{dE} / \mathrm{dx}$ in the calorimeter versus rigidity: The isotopic analysis of nuclei with the calorimeter was restricted to events that do not interact inside the calorimeter. To check if an interaction occurs, in each silicon layer the total energy detected $q_{t o t}$ and the energy deposited in the strip closest to the track and the neighboring strip on each side $q_{\text {track }}$ were derived. Starting from the top of the calorimeter the $\Sigma q_{\text {track }} / \Sigma q_{\text {tot }}$ at each layer was calculated. As long as this value was greater than 0.9 , the layers were used for further analysis. A value of 0.9 was chosen since it was found to give a good compromise between high efficiency and rejection of interactions. In the single silicon layer, the energy loss distributions show a Landau tail that degrades the resolution of the $\mathrm{dE} / \mathrm{dx}$ measurement. Using a truncation method, the $50 \%$ of samples with larger pulse amplitudes were excluded before taking the mean of the $\mathrm{dE} / \mathrm{dx}$ measurements, thus reducing the effect of the Landau tail. An energy dependent lower limit on the number of layers after the 50\% truncation was imposed. For more details of the selection criteria see [6].

The isotope separation and the determination of isotope fluxes were performed identically to [6] in intervals of kinetic energy per nucleon. Since the magnetic spectrometer measures the rigidity of particles and not the kinetic energy, this means that different rigidity intervals have to be 

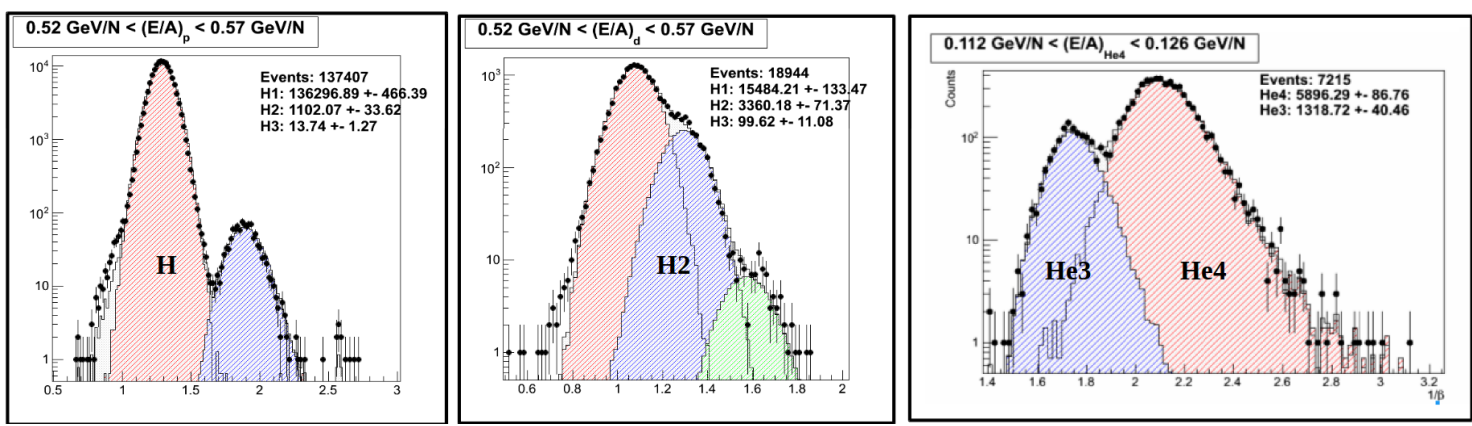

Figure 5: Likelihood fit applied to a sample particle selected with the ToF. Left panel: ${ }^{1} \mathrm{H}$ selection. Central panel: ${ }^{2} \mathrm{H}$ selection. Right panel: ${ }^{4} \mathrm{He}$ selection.

analyzed depending on the mass of the isotope under study.

To get the raw isotope numbers, a likelihood fit (the "TFractionFitter" method in ROOT [7]) was performed to the $1 / \beta$ and $\mathrm{dE} / \mathrm{dx}$ distributions of the selected events. The likelihood fit needs a model distribution to create the expected probability density function, the full Monte Carlo simulation of the PAMELA apparatus based on the GEANT4 was used for this task. Figure 5 describes the fit procedure for three samples of events using the ToF. The left panel shows the $1 / \beta$ distribution for ${ }^{1} \mathrm{H}$ energies between 0.52 and $0.57 \mathrm{GeV} / \mathrm{n}$, with the data points in black, and the red and blue areas showing the estimated individual ${ }^{1} \mathrm{H}$ and ${ }^{2} \mathrm{H}$ signals. The central panel of Figure 5 shows $1 / \beta$ distribution for ${ }^{2} \mathrm{H}$ energies between 0.52 and $0.57 \mathrm{GeV} / \mathrm{n}$, with the red, blue, and green areas showing the estimated individual ${ }^{1} \mathrm{H},{ }^{2} \mathrm{H}$, and ${ }^{3} \mathrm{H}$ signals (the ${ }^{3} \mathrm{H}$ is locally produced in the instrument). Note that in this figure the ${ }^{1} \mathrm{H}$ contribution is suppressed using the energy loss measurements in the silicon layers of the tracking system and in the scintillators of the ToF versus the rigidity, for details of this suppression method see [6]. The right panel of Figure 5 represent the isotopic separation of ${ }^{3} \mathrm{He}$ (blue area) and ${ }^{4} \mathrm{He}$ (red area)between for ${ }^{4} \mathrm{He}$ energies between 0.112 and $0.126 \mathrm{GeV} / \mathrm{n}$.

With these procedures was possible to derive the numbers of ${ }^{1} \mathrm{H}$ and ${ }^{2} \mathrm{H}$ from 0.12 up to $0.57 \mathrm{GeV} / \mathrm{n}$ with the ToF and from 0.23 up to $1.1 \mathrm{GeV} / \mathrm{n}$ with the calorimeter. The ${ }^{3} \mathrm{He}$ and ${ }^{4} \mathrm{He}$ separation was possible between 0.12 and $0.87 \mathrm{GeV} / \mathrm{n}$ with the ToF and from 0.25 up to $1.37 \mathrm{GeV} / \mathrm{n}$ with the calorimeter. For more details of the isotopic separation see [6].

\section{Flux calculation}

The absolute isotope flux $\Phi(E)$ in kinetic energy were obtained as follows:

$$
\Phi(E)=\frac{N(E)}{G(E) \times T \times \varepsilon(E) \times \Delta E}
$$

where $N(E)$ is the count distribution, $\varepsilon(E)$ the efficiencies of the tracking system selections, $G(E)$ the geometrical factor, $T$ the live-time and $\Delta E$ the width of the energy interval.

When possible, the efficiencies were evaluated with flight data exploiting the detector redundancy to select clean samples of isotopes. Simulated data were used to cross check the flight 


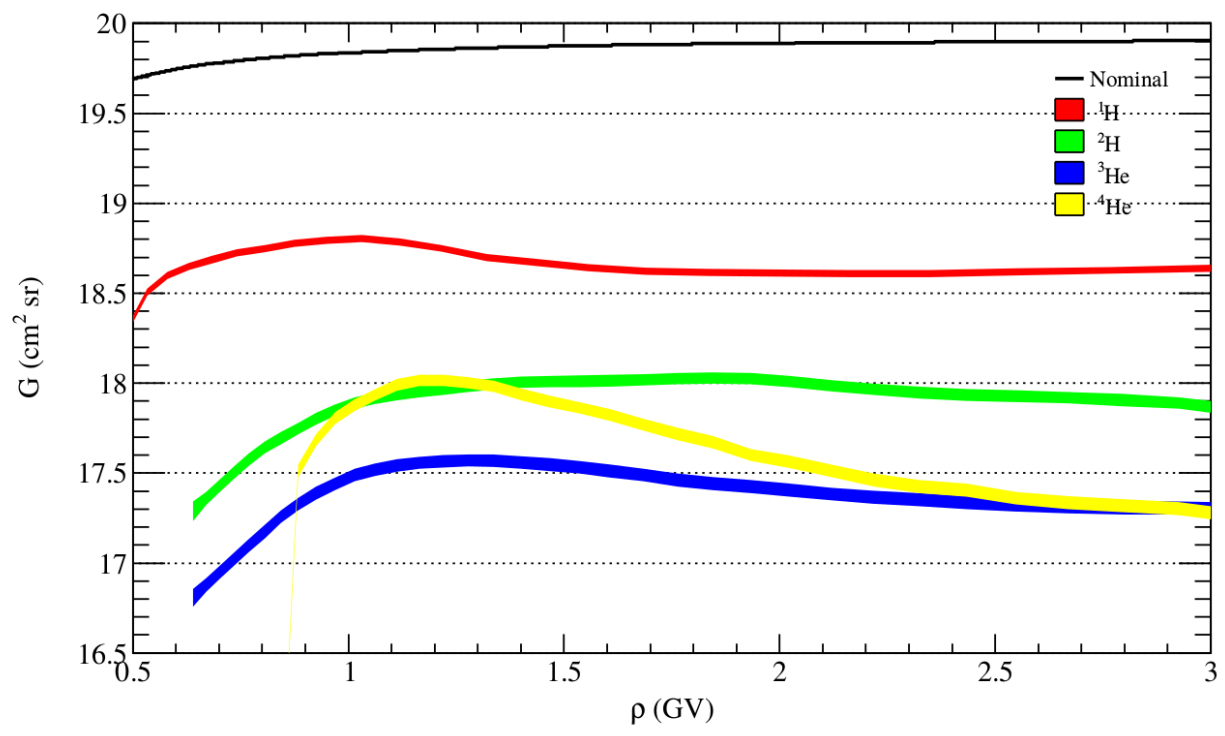

Figure 6: Nominal geometrical factor $G(E)$ (black line) and the effective geometrical factor $\mathrm{G}$ for the four different isotopes under study as a function of rigidity.

efficiency in order to emphasize possible contamination of the flight sample. When the flight samples resulted contaminated the simulated efficiency were used. The tracker efficiency was entirely evaluated using simulated data.

Figure 6 shows the nominal geometrical factor $G(E)=19.9 \mathrm{~cm}^{2}$ sr (black line) which was corrected in order to account for the hydrogen and helium nuclei which are lost due to hadronic interactions in the aluminum pressurized container $(2 \mathrm{~mm}$ thick) and the top scintillators. Moreover the ${ }^{2} \mathrm{H}$ and ${ }^{3} \mathrm{He}$ geometrical factor were corrected for the locally produce contamination from fragmentation of higher nuclei in the $2 \mathrm{~mm}$ aluminum dome. These corrections were evaluated with the Monte Carlo simulation. The contribution in the ${ }^{3} \mathrm{He}$ sample from ${ }^{4} \mathrm{He}$ fragmentation was found to be less than $1 \%$ while the contamination of ${ }^{2} \mathrm{H}$ was found to be around $10 \%$ at $0.1 \mathrm{GeV} / \mathrm{n}$ decreasing at $1 \%$ around $0.6 \mathrm{GeV} / \mathrm{n}$.

Finally, due to the finite spectrometer resolution and the ionization energy losses, the measured rigidity with the tracker system differs from the initial energies at the top of the payload; to overcome these effects an unfolding procedure was applied, e.g. see [9].

\section{Conclusion}

The analysis of the time dependent flux of the Helium and Hydrogen isotopes performed with the PAMELA instrument has been presented. Data acquired between July 2006 and September 2014 were used to measured the yearly average fluxes for a total of 9 spectra. These results will be very important to test and calibrate models used to described the propagation of CR inside the Heliosphere as well as to perform a better determination of their local interstellar spectra. Preliminary results for ${ }^{1} \mathrm{H},{ }^{2} \mathrm{H},{ }^{3} \mathrm{He}$ and ${ }^{4} \mathrm{He}$ fluxes will be presented at the ICRC oral session. 


\section{Acknowledgments}

South African authors acknowledge the partial financial support of the SA National Research Foundation.

\section{References}

[1] Potgieter, Living Rev Solar Physics, 10, 3, 2013

[2] Potgieter, et. al., Solar Physics, 289, 391, 2014

[3] Adriani, O., et. al., ApJ, 765, 91, 2013

[4] Adriani, O., et. al., ApJ Letters, 854,L2, 2018

[5] Adriani, O., et. al., ApJ, 810, 142, 2015

[6] Adriani, O., et. al., ApJ, 818, 68, 2016

[7] Brun, R. \& Rademakers, F, Nucl. Instrum. Meth. A 389, 81, 1997

[8] Picozza, P., et al., ApJ, 27, 296, 2007

[9] D'Agostini, G. Nuclear Instruments and Methods in Physics Research A, 362, 487, 1995 